\title{
Stellungnahme zum Eckpunktepapier des BMI „Offenes Regierungs- und Verwaltungshandeln (Open Government)"
}

Die Beauftragten für Informationsfreiheit des Bundes und der Länder begrüßen die Vorlage des Eckpunktepapiers „Open Government ${ }^{\text {"1 }}$ durch das Bundesministerium des Innern. Der Einsatz der Informationstechnik bietet die große Chance, Transparenz und Informationsfreiheit auf der Ebene des Bundes, der Länder und der Kommunen einen großen Schritt voran zu bringen.

Die Entwicklung des Eckpunktepapiers ist noch nicht abgeschlossen. Wir regen hier einige Verbesserungen an, die im Folgenden den einzelnen Abschnitten zugeordnet werden.

Open Government bedeutet Kulturwandel

Die Aussagen über einen Wandel der Verwaltungskultur und ein verändertes Zusammenspiel von Gesellschaft und Staat weisen in die richtige Richtung. Die Informationsfreiheitsbeauftragten empfehlen, an dieser Stelle den Leitgedanken einer Überwindung des überkommenen Amtsgeheimnisses noch deutlicher zu formulieren. Die Informationsfreiheitsgesetze des Bundes und der Länder sind um verbindliche Verpflichtungen aller öffentlichen Stellen zu erweitern, von sich aus Informationen zu veröffentlichen. Wir verweisen hier auf die Entschließung der 21. Konferenz der Informationsfreiheitsbeauftragten vom 13. Dezember 2010 „Open Data: Mehr statt weniger Transparenz".

Gesetzliche Regelungen zur proaktiven Veröffentlichungspflicht sind auch in den Bundesländern erforderlich, die noch nicht über Informationsfreiheitsgesetze verfügen (BadenWürttemberg, Bayern, Hessen, Niedersachsen und Sachsen).

Die Bundesregierung und die Regierungen der Bundesländer sollten sich dazu verpflichten, Kabinettvorlagen und Referentenentwürfe frühzeitig ins Netz zu stellen und damit der öffentlichen Diskussion zugänglich zu machen. Es sollte selbstverständlich werden, bei wichtigen Gesetzentwürfen Diskussionsplattformen einzurichten. So kann eine aktivere Teilhabe der Bürgerinnen und Bürger erfolgen.

Bestehende Ansätze nutzen und ausbauen

Der knapp gehaltene Hinweis auf bestehende Initiativen und Informationsforen greift zu kurz. Nötig ist vielmehr eine verbindliche Regelung, dass amtliche Informationen über originär staatliche Plattformen zur Verfügung gestellt werden. Das Zugänglichmachen von Informationen über soziale Netzwerke wie z.B. Facebook genügt diesen Transparenzanforderungen nicht.

Den Rechtsrahmen überprüfen und einheitliche Anwendung empfehlen

Der Verweis auf die bereits bestehenden Rechtsgrundlagen genügt nicht. Die Analyse des bestehenden Rechtsrahmens darf

1 http://www.verwaltung-innovativ.de/nn_1978620/SharedDocs/Publikationen/Regierungsprogramm/strategiepapier_,templateld=raw, property=publica tionFile.pdf/strategiepapier_.pdf nicht bedeuten, dass bereits erreichte Standards wieder zurückgesetzt werden. Insbesondere sollten die Informationsfreiheitsgesetze als wesentlicher Bestandteil des bestehenden Rechtsrahmens an dieser Stelle Erwähnung finden.

Unverbindlich sind auch die Formulierungen zu den Nutzungsbestimmungen. Der Staat sollte nicht selbst Lizenzgeber sein. Dies widerspricht dem Grundgedanken von Open Data. Mit öffentlichen Mitteln erhobene Datenbestände dürfen nicht durch Urheber- oder Nutzungsbeschränkungen blockiert werden. Das Urheberrecht kann nur in den Fällen, in denen Werke Dritter Bestandteil öffentlicher Informationen werden, einer Veröffentlichung entgegenstehen. Mit öffentlichen Mitteln erhobene Datenbestände dürfen durch weitergehende Nutzungsbeschränkungen nicht blockiert werden.

Bedauerlich ist, dass die in der Entwurfsfassung des Eckpunktepapiers vom 20. Juni 2011 unter Nr. 5 vorgesehene „Einfache Kostenregelung, um Anreize zur Verwendung von Daten zu geben“, in der Neufassung des Papiers nicht mehr vorkommt. Die alte Formulierung weist in die richtige Richtung: „Eine kostenlose Abgabe von Daten fördert die Weiterverwendung von Daten und kann über Innovation und den Aufbau von Geschäftsmodellen wirtschaftliche Impulse setzen, die zu neuen staatlichen Einnahmequellen führen."

Die Informationsfreiheitsbeauftragten vertreten den Standpunkt, dass öffentliche Daten von den zuständigen öffentlichen Stellen grundsätzlich kostenfrei zur Verfügung gestellt werden müssen. Dabei darf es keine Rolle spielen, welche Absicht mit der Datenverwendung verbunden ist. Durch die Erhebung der Verwendungsabsicht darf die Voraussetzungslosigkeit des Zugangs zu Informationen nicht durch die Hintertür ausgehebelt werden. Niemand soll sich für sein Informationsinteresse rechtfertigen müssen. So sehen es auch die bereits bestehenden Informationsfreiheitsgesetze vor.

Die in der Entwurfsfassung vom 6. September 2011 vorgesehene Prüfung der Geldleistungsregelungen für die Nutzung maschinenlesbarer Daten bleibt weit hinter den früheren Ansätzen zurück. Sie wird den Anforderungen an eine moderne Informationspolitik nicht gerecht.

Bund und Länder sollten die Pläne der für die digitale Agenda zuständigen EU Kommissarin Neelie Kroes unterstützen (Vorschlag für eine Richtlinie des Europäischen Parlaments und des Rates zur Änderung der Richtlinie 2003/98 über die Weiterverwendung von Informationen des öffentlichen Sektors (KOM (2011) 877 endgültig). Danach dürfen künftig Informationen, die von öffentlichen Stellen zugänglich gemacht werden, zu beliebigen Zwecken weiterverwendet werden, soweit nicht Urheberrechte Dritter zu schützen sind.

Konferenz der Informationsfreiheitsbeauftragten in Deutschland (IFK) 